\title{
Hard Lefschetz properties and distribution of spectra in singularity theory and Ehrhart theory
}

\author{
Antoine Douai* \\ Université Côte d'Azur, CNRS, LJAD, FRANCE \\ Email address: antoine.douai@univ-cotedazur.fr
}

March 10, 2021

\begin{abstract}
Motivated by the distribution of spectra in singularity theory and combinatorics, we study a hard Lefschetz property for Laurent polynomials and for polytopes and we give combinatorial criteria for this property to be true. This also provides informations about a conjecture by Katzarkov-Kontsevich-Pantev.
\end{abstract}

\section{Introduction}

Let $P$ be a lattice polytope in $\mathbb{R}^{n}$ (the convex hull of a finite set in $N:=\mathbb{Z}^{n}$ ). Define, for a positive integer $\ell, L_{P}(\ell):=\operatorname{Card}((\ell P) \cap N)$. Then $L_{P}$ is a polynomial in $\ell$ of degree $n$, the Ehrhart polynomial of $P$ and

$$
1+\sum_{m \geq 1} L_{P}(m) z^{m}=\frac{\delta_{0}+\delta_{1} z+\cdots+\delta_{n} z^{n}}{(1-z)^{n+1}}
$$

where the $\delta_{j}$ 's are nonnegative integers. The vector $\delta_{P}=\left(\delta_{0}, \cdots, \delta_{n}\right)$ is called the $\delta$-vector of the polytope $P$. The first result in the study of the distribution of the $\delta$-vector is probably Hibi's symmetry property $\delta_{i}=\delta_{n-i}$ for $i=1, \cdots, n$, which is actually a characterization of reflexive polytopes [11. The second one concerns the unimodality of the $\delta$-vector of a reflexive polytope: taking into account the previous symmetry property, one could expect $\delta_{0} \leq \delta_{1} \leq \cdots \leq \delta_{[n / 2]}$ and $\delta_{[n / 2]} \geq \delta_{[n / 2]+1} \geq \cdots \geq \delta_{n}$. This is indeed what happens in dimension less than or equal to five [10], but this unimodality may fail in dimension greater than or equal to six, see for instance [20, Example 3.4], [15, [16.

On the other hand, singularity theory meets Ehrhart theory by the means of the $\delta$-vector: the spectrum at infinity of a tame Laurent polynomial determines the $\delta$-vector of its Newton polytope and both coincide if the latter is reflexive [5]. This interplay encourages us also to study the unimodality (and more generally, the distribution) of the spectrum at infinity of a regular function.

Classically, unimodality can be seen as a combinatorial application of the hard Lefschetz theorem (see [19] for instance where it is shown that the Poincaré polynomial of a smooth complex projective variety is unimodal) and we are naturally led to study a hard Lefschetz property for regular functions (singularity side) and for polytopes (Ehrhart theory side). On the singularity side, the hard Lefschetz property for a Laurent polynomial $f$ is provided by the multiplication by $f$ on a graded Jacobi ring. The hard Lefschetz property for a simplicial polytope $P$ is provided by the hard Lefschetz property for the orbifold cohomology of the orbifold associated with $P$ by the work of Borisov, Chen and Smith [2]. Both are related by a mirror theorem. This is detailed in Section 3, where we also give a combinatorial criterion for these hard Lefschetz

${ }^{*}$ Mathematics Subject Classification 52B20, 32S40, 14J33. Key words and phrases: toric varieties, hard Lefschetz properties, spectrum of regular functions and polytopes, mirror theorem, orbifold cohomology, distribution of spectral numbers 
properties to be satisfied: let $P$ be a full dimensional, simplicial, lattice polytope in $\mathbb{R}^{n}$ containing the origin as an interior point and let $\Sigma_{P}$ be the fan over the proper faces of $P$. For a $n$-dimensional cone $\sigma \in \Sigma_{P}$, let $\operatorname{Box}(\sigma)$ be the set of $v \in N$ such that $v=\sum_{\rho_{i} \subset \sigma} q_{i} b_{i}$ for some $0 \leq q_{i}<1$, where $\rho_{i}$ denotes the ray generated by the vertex $b_{i}$ of $P$. We have the following generalization of [8, Proposition 4.1] (see Proposition [3.9):

Theorem 1.1 The polytope P satisfies the hard Lefschetz property if and only if

$$
[\nu(v)]=(\operatorname{dim} \sigma(v)-1) / 2 \text { if } \nu(v) \notin \mathbb{N}
$$

and

$$
\nu(v)=\operatorname{dim} \sigma(v) / 2 \text { if } \nu(v) \in \mathbb{N}
$$

for all $v \in \cup_{\sigma} \operatorname{Box}(\sigma)$ (the union is taken over all the $n$-dimensional cones of $\Sigma_{P}$ ), where $\sigma(v)$ denotes the smallest cone of $\Sigma_{P}$ containing $v$ and $\nu$ is the Newton function of $P$.

When applied to a reduced simplex $\Delta$, this criterion reduces to an arithmetic condition on its weight, see Proposition 4.1 (the weight of a simplex $\Delta:=\operatorname{conv}\left(v_{0}, \cdots, v_{n}\right)$ is the tuple $\left(q_{0}, \cdots, q_{n}\right)$, arranged by increasing order, where $q_{i}:=\left|\operatorname{det}\left(v_{0}, \cdots, \widehat{v_{i}}, \cdots, v_{n}\right)\right|$ and the simplex $\Delta$ is reduced if $\operatorname{gcd}\left(q_{0}, \cdots, q_{n}\right)=1$, see Section 4.1 for details). For instance, if moreover $\Delta$ is reflexive, that is if $q_{i}$ divides $\mu:=q_{0}+\cdots+q_{n}$ for $i=0, \cdots, n$, we get:

Proposition 1.2 Assume that the reduced and reflexive simplex $\Delta$ of weight $\left(q_{0}, \cdots, q_{n}\right)$ satisfies the hard Lefschetz property. Then,

$$
\frac{2 \mu}{q_{n}}=n+1+m\left(q_{n}\right)
$$

where $m\left(q_{n}\right)$ denotes the multiplicity of $q_{n}$ in the ordered tuple $\left(q_{0}, \cdots, q_{n}\right)$.

See Remark 4.4. For example, (2) fails for the three dimensional reflexive and reduced simplex $\Delta$ of weight $(1,1,1,3)$ : this simplex does not satisfy the hard Lefschetz property. Actually, we have a stronger statement (a necessary and sufficient condition, see Corollary 4.3) and it follows from our computations that the hard Lefschetz properties are not common at all (and this answers a question in [8, Section 4]): for instance, we check that the hard Lefschetz property is true for 2 out the 14 three dimensional reduced and reflexive simplices described in 4 .

This has an interpretation in Hodge theory: it has been noticed in [17 that a Laurent polynomial $f$ satisfies the hard Lefschetz property if and only if the mixed Hodge structure produced by the Laplace transform of its Gauss-Manin system is of Hodge-Tate type. As a consequence, we get informations about a conjecture by Katzarkov-Kontsevich-Pantev [12, Conjecture 3.6], see Proposition 4.7 it turns out that only a few Laurent polynomials whose Newton polytopes are reduced and reflexive simplices satisfy this conjecture.

Last, and this was after all our starting point, the hard Lefschetz properties studied in this paper are related with the unimodality of the spectrum at infinity of a regular function. This is discussed in Section 5 .

These notes were motivated by Sabbah's paper [17, in which the "smooth" case is considered.

\section{Spectra}

In this section, we recall some results from [5]. Let $N$ be the lattice $\mathbb{Z}^{n}$ and let $P \subset N_{\mathbb{R}}$ be a full dimensional lattice polytope containing the origin as an interior point. We assume throughout this paper that $P$ is simplicial.

Let $\Sigma_{P}$ be the (simplicial) fan in $N_{\mathbb{R}}$ obtained by taking the cones over the proper faces of $P$ and let $X_{\Sigma_{P}}$ be the complete, projective, toric variety of the fan $\Sigma_{P}$. The Newton function of $P$ is the function $\nu: N_{\mathbb{R}} \rightarrow \mathbb{R}$ which takes the value 1 at the vertices of $P$ and which is linear on each cone of $\Sigma_{P}$. The Milnor 
number of $P$ is $\mu_{P}:=n ! \operatorname{vol}(P)$ where the volume $\operatorname{vol}(P)$ is normalized such that the volume of the cube is equal to 1 . We define the Newton spectrum of $P$ by

$$
\operatorname{Spec}_{P}(z):=(1-z)^{n} \sum_{v \in N} z^{\nu(v)} .
$$

Let $f(u)=\sum_{m \in \mathbb{Z}^{n}} a_{m} u^{m}$ be a Laurent polynomial defined on $\left(\mathbb{C}^{*}\right)^{n}$. The Newton polytope $P$ of $f$ is the convex hull of supp $f:=\left\{m \in \mathbb{Z}^{n}, a_{m} \neq 0\right\}$ in $\mathbb{R}^{n}$. We assume in this text that $f$ is convenient (its Newton polytope contains the origin as an interior point) and nondegenerate in the sense of Kouchnirenko 13. Let $\mathcal{A}_{f}:=\mathcal{B} / \mathcal{L}$ where $\mathcal{B}:=\mathbb{C}\left[u_{1}, u_{1}^{-1}, \cdots, u_{n}, u_{n}^{-1}\right]$ and $\mathcal{L}:=\left(u_{1} \frac{\partial f}{\partial u_{1}}, \cdots, u_{n} \frac{\partial f}{\partial u_{n}}\right)$ is the ideal generated by the partial derivative $u_{1} \frac{\partial f}{\partial u_{1}}, \cdots, u_{n} \frac{\partial f}{\partial u_{n}}$ of $f$. We define an increasing filtration $\mathcal{N}_{\bullet}$ on $\mathcal{B}$, indexed by $\mathbb{Q}$, by setting

$$
\left.\left.\mathcal{N}_{\alpha} \mathcal{B}:=\left\{g \in \mathcal{B}, \operatorname{supp}(g) \in \nu^{-1}(]-\infty ; \alpha\right]\right)\right\}
$$

where $\nu$ is the Newton function of the Newton polytope $P$ of $f$ and $\operatorname{supp}(g)=\left\{m \in \mathbb{N}^{n}, a_{m} \neq 0\right\}$ if $g=\sum_{m \in \mathbb{N}^{n}} a_{m} u^{m} \in \mathcal{B}$. By projection, the Newton filtration $\mathcal{N}_{\bullet}$ on $\mathcal{B}$ induces the Newton filtration $\mathcal{N}_{\bullet}$ on $\mathcal{A}_{f}$ and the spectrum at infinity of $f$ is given by

$$
\operatorname{Spec}_{f}(z)=\sum_{\alpha \in \mathbb{Q}} \operatorname{dim}_{\mathbb{C}}\left(\operatorname{gr}_{\alpha}^{\mathcal{N}} \mathcal{A}_{f}\right) z^{\alpha} .
$$

Both spectra are related: if $f$ is a convenient and nondegenerate Laurent polynomial with Newton polytope $P$, we have $\operatorname{Spec}_{f}(z)=\operatorname{Spec}_{P}(z)$, see [5, Corollary 2.2].

We are interested in the distribution of $\operatorname{Spec}_{f}(z)$ and $\operatorname{Spec}_{P}(z)$ and it will be useful to decide when these spectra are polynomials. Recall that a lattice polytope $P$ is reflexive if it contains the origin as an interior point and if its polar polytope $P^{\circ}:=\left\{y \in M_{\mathbb{R}},\langle y, x\rangle \leq 1\right.$ for all $\left.x \in P\right\}$ is a lattice polytope.

Proposition 2.1 [5, Proposition 5.1] The following are equivalent:

1. $\operatorname{Spec}_{P}(z)$ is a polynomial,

2. $P$ is reflexive,

3. $\operatorname{Spec}_{P}(z)=\delta_{0}+\delta_{1} z+\cdots+\delta_{n} z^{n}$ where $\left(\delta_{0}, \cdots, \delta_{n}\right)$ is the $\delta$-vector of $P$.

On the singularity side, we get (and we will refer to this case as the unipotent case):

Corollary 2.2 Let $f$ be a convenient and nondegenerate Laurent polynomial. Then its spectrum at infinity $\operatorname{Spec}_{f}(z)$ is a polynomial if and only if its Newton polytope $P$ is reflexive.

\section{The hard Lefschetz property for Laurent polynomials and poly- topes}

Let $f$ be a convenient and nondegenerate Laurent polynomial defined on $\left(\mathbb{C}^{*}\right)^{n}$. The multiplication by $f$ induces maps

$$
[f]: \operatorname{gr}_{\alpha}^{\mathcal{N}} \mathcal{A}_{f} \longrightarrow \mathrm{gr}_{\alpha+1}^{\mathcal{N}} \mathcal{A}_{f}
$$

for $\alpha \in \mathbb{Q}$. The following definition can already be found in [17]:

Definition 3.1 Let $f$ be a convenient and nondegenerate Laurent polynomial on $\left(\mathbb{C}^{*}\right)^{n}$. We will say that $f$ satisfies the hard Lefschetz property $(H L)$ if the multiplication by $f$ induces isomorphisms

$$
[f]^{n-1-2 k}: \operatorname{gr}_{\alpha+k}^{\mathcal{N}} \mathcal{A}_{f} \stackrel{\cong}{\longrightarrow} \operatorname{gr}_{\alpha+n-1-k}^{\mathcal{N}} \mathcal{A}_{f}
$$


for $0 \leq k \leq[(n-1) / 2]$ and $\alpha \in] 0,1[$ and

$$
[f]^{n-2 k}: \operatorname{gr}_{k}^{\mathcal{N}} \mathcal{A}_{f} \stackrel{\cong}{\longrightarrow} \operatorname{gr}_{n-k}^{\mathcal{N}} \mathcal{A}_{f}
$$

for $0 \leq k \leq[n / 2]$.

Let now $P$ be a simplicial full-dimensional lattice polytope in $\mathbb{R}^{n}$ containing the origin as an interior point. We will denote by $\mathcal{V}(P):=\left\{b_{1}, \cdots, b_{r}\right\}$ the set of its vertices. Let

- $\mathcal{X}$ be the Deligne-Mumford stack associated with the stacky fan $\boldsymbol{\Sigma}:=\left(\mathbb{Z}^{n}, \Sigma_{P}, \mathcal{V}(P)\right)$ by [2, Section 3],

- $I_{\mathcal{X}}=\coprod_{\ell \in F} \mathcal{X}_{\ell}$ be the decomposition into connected components of the inertia orbifold of $\mathcal{X}$, see 1 , Section 4.1],

- $H_{\text {orb }}^{2 \alpha}(\mathcal{X}, \mathbb{C}):=\oplus_{\ell \in F} H^{2\left(\alpha-\operatorname{age}\left(\mathcal{X}_{\ell}\right)\right)}\left(\mathcal{X}_{\ell}, \mathbb{C}\right)$ be the orbifold cohomology groups of $\mathcal{X}$, where age $\left(\mathcal{X}_{\ell}\right)$ the age of the sector $\mathcal{X}_{\ell}$, see [1, Definition 4.8],

- $f_{P}$ be the Laurent polynomial on $\left(\mathbb{C}^{*}\right)^{n}$ defined by $f_{P}(u):=\sum_{b \in \mathcal{V}(P)} u^{b}$.

See [2, Proposition 4.7] for a toric description of the sectors $\mathcal{X}_{\ell}$.

The following wonderful result is due to [2, with a little help from [13] (the orbifold cohomology is equipped with the orbifold cup-product $\cup_{\text {orb }}$, see [2, Section 6]).

Proposition $3.2[2]$ There is an isomorphism of $\mathbb{Q}$-graded rings

$$
\varphi: H_{\text {orb }}^{2 *}(\mathcal{X}, \mathbb{C}) \stackrel{\cong}{\longrightarrow} \operatorname{gr}_{*}^{\mathcal{N}} \mathcal{A}_{f_{P}}
$$

Proof. Notice first that $f_{P}$ is convenient (because $P$ contains the origin as an interior point) and nondegenerate (thanks to the simpliciality assumption) with respect to its Newton polytope $P$. By [13, Théorème 4.1], the map $\partial: \mathbb{C}\left[u, u^{-1}\right]^{n} \rightarrow \mathbb{C}\left[u, u^{-1}\right]$ defined by $\partial\left(b_{1}, \cdots, b_{n}\right)=b_{1} u_{1} \frac{\partial f}{\partial u_{1}}+\cdots+b_{n} u_{n} \frac{\partial f}{\partial u_{n}}$ is strict with respect to the Newton filtration. Hence, and by the definition of the Newton filtration, the graded ring $\operatorname{gr}_{*}^{\mathcal{N}} \mathcal{A}_{f_{P}}$ is nothing but the "Stanley-Reisner presentation" of $\mathcal{X}$ given by the right hand side of [2, Theorem 1.1] and the result follows from loc. cit.

It should be emphasized that Proposition 3.2 provides an isomorphism of rings, and this really depends on the special form of $f_{P}$, from which we also get $\varphi^{-1}\left(\left[f_{P}\right]\right) \in H^{2}\left(\mathcal{X}_{0}, \mathbb{C}\right)$ where $\mathcal{X}_{0}$ denotes the untwisted sector.

The cohomology $H^{*}\left(\mathcal{X}_{\ell}, \mathbb{C}\right)$ of the twisted sector $\mathcal{X}_{\ell}$ is a $H^{*}\left(\mathcal{X}_{0}, \mathbb{C}\right)$-module under the orbifold cup-product, and this module structure is basically given by the standard cup-product on $H^{*}\left(\mathcal{X}_{\ell}, \mathbb{C}\right)$, see for instance 8 , Proposition 3.2], [2, Proof of Theorem 1.1]. We define, for $\omega \in H^{2}\left(\mathcal{X}_{0}, \mathbb{C}\right)$,

$$
L_{\omega}(\eta):=\pi_{0}^{*} \omega \cup_{\text {orb }} \eta
$$

where $\pi_{0}$ denotes the restriction of $\pi: I_{\mathcal{X}} \longrightarrow \mathcal{X}$ to the non-twisted sector. We have the following counterpart of Definition 3.1 ,

Definition 3.3 We will say that $P$ satisfies the hard Lefschetz property $(H L)$ if there exists $\omega \in H^{2}\left(\mathcal{X}_{0}, \mathbb{C}\right)$ such that the orbifold cup-product by $\omega$ induces isomorphisms

$$
L_{\omega}^{n-1-2 k}: H_{\text {orb }}^{2(\alpha+k)}(\mathcal{X}, \mathbb{C}) \stackrel{\cong}{\longrightarrow} H_{\text {orb }}^{2(\alpha+n-1-k)}(\mathcal{X}, \mathbb{C})
$$

for $0 \leq k \leq[(n-1) / 2]$ and $\alpha \in] 0,1[$ and

$$
L_{\omega}^{n-2 k}: H_{\mathrm{orb}}^{2 k}(\mathcal{X}, \mathbb{C}) \stackrel{\cong}{\longrightarrow} H_{\mathrm{orb}}^{2(n-k)}(\mathcal{X}, \mathbb{C})
$$

for $0 \leq k \leq[n / 2]$. 
Remark 3.4 Assume that $P$ is a (reduced) simplex, as in Section 4.1 below (and we will mainly consider this situation). The corresponding orbifold is a weighted projective space $\mathbb{P}(w)$. We have $\operatorname{dim} H^{2}\left(\mathcal{X}_{0}, \mathbb{C}\right)=1$ and this vector space is generated by the Chern class $\omega:=c_{1}\left(\mathcal{O}_{\mathbb{P}(w)}(1)\right.$ ) (see [14, Proposition 3.6 and Remark 3.7 ] for the definition of $\mathcal{O}_{\mathbb{P}(w)}(1)$ and its restrictions to the various sectors). The action of $L_{\omega}$ is then computed using Corollary 3.18 of loc. cit.

We now give criteria for this hard Lefschetz property to be true. Let $P$ be a simplicial full dimensional lattice polytope in $\mathbb{R}^{n}$ containing the origin as an interior point and let $\mathcal{X}$ as above. For $\ell \in F$, we put $\ell^{-1}:=I(\ell)$ where $I$ is the involution on $F$ induced by the involution on the inertia orbifold $I_{\mathcal{X}}=\coprod_{\ell \in F} \mathcal{X}_{\ell}$ defined in [1, (4.3)]. We will denote by $[x]$ the integral part of $x$. First, we have the following generalization of a result of Fernandez [8]:

Theorem 3.5 The polytope $P$ satisfies (HL) if and only if

$$
\left[\operatorname{age}\left(\mathcal{X}_{\ell}\right)\right]=\left[\operatorname{age}\left(\mathcal{X}_{\ell^{-1}}\right)\right]
$$

for all $\ell \in F$.

Proof. In what follows, we put $i_{\ell}:=\operatorname{age}\left(\mathcal{X}_{\ell}\right)$. Assume first that $P$ satisfies the hard Lefschetz property (8). Let $\alpha \in] 0,1\left[\right.$. Because the orbifold cup-product by $\omega \in H^{2}\left(\mathcal{X}_{0}, \mathbb{C}\right)$ preserves the cohomology of each sector $\mathcal{X}_{\ell}$, we get the isomorphisms

$$
L_{\omega}^{n-1-2 k}: H^{2\left(\alpha+k-i_{\ell}\right)}\left(\mathcal{X}_{\ell}, \mathbb{C}\right) \stackrel{\cong}{\longrightarrow} H^{2\left(\alpha+n-1-k-i_{\ell}\right)}\left(\mathcal{X}_{\ell}, \mathbb{C}\right) .
$$

for $\ell \in F$ and $k \leq[(n-1) / 2]$, where $L_{\omega}(\eta)=\pi_{\ell}^{*} \omega \wedge \eta \in H^{q+2}\left(\mathcal{X}_{\ell}, \mathbb{C}\right)$ if $\eta \in H^{q}\left(\mathcal{X}_{\ell}, \mathbb{C}\right)$ and $\pi_{\ell}$ denotes the restriction of $\pi$ to $\mathcal{X}_{\ell}$. Since (11) is relevant only if $\alpha-i_{\ell} \in \mathbb{Z}$, we may assume that $\alpha=i_{\ell}-\left[i_{\ell}\right]$.

By [1, Lemma 4.6], we have $n_{\ell}:=\operatorname{dim} \mathcal{X}_{\ell}=n-i_{\ell}-i_{\ell^{-1}}$ and it follows that the isomorphisms (11) are equivalent to

$$
L_{\omega}^{n-1-2 k}: H^{2\left(k-\left[i_{\ell}\right]\right)}\left(\mathcal{X}_{\ell}, \mathbb{C}\right) \stackrel{\cong}{\longrightarrow} H^{2\left(n_{\ell}-1-k+i_{\ell}+i_{\ell^{-1}}-\left[i_{\ell}\right]\right)}\left(\mathcal{X}_{\ell}, \mathbb{C}\right) .
$$

Because $i_{\ell}+i_{\ell^{-1}} \in \mathbb{Z}$ and $i_{\ell} \notin \mathbb{Z}$, we have $i_{\ell}+i_{\ell^{-1}}=\left[i_{\ell}\right]+\left[i_{\ell^{-1}}\right]+1$ and we finally get the isomorphisms

$$
L_{\omega}^{n-1-2 k}: H^{2\left(k-\left[i_{\ell}\right]\right)}\left(\mathcal{X}_{\ell}, \mathbb{C}\right) \stackrel{\cong}{\longrightarrow} H^{2\left(n_{\ell}-k+\left[i_{\ell}-1\right]\right)}\left(\mathcal{X}_{\ell}, \mathbb{C}\right) .
$$

Since $i_{\ell}+i_{\ell^{-1}} \leq n$, we may assume that $\left[i_{\ell}\right] \leq[(n-1) / 2]$ and we can put $k=\left[i_{\ell}\right]$ in (12) in order to get the isomorphism

$$
H^{0}\left(\mathcal{X}_{\ell}, \mathbb{C}\right) \stackrel{\cong}{\longrightarrow} H^{2\left(n_{\ell}-\left[i_{\ell}\right]+\left[i_{\ell-1}\right]\right)}\left(\mathcal{X}_{\ell}, \mathbb{C}\right) .
$$

It follows that $\left[i_{\ell^{-1}}\right]-\left[i_{\ell}\right] \leq 0$. In particular, we have also $\left[i_{\ell^{-1}}\right] \leq[(n-1) / 2]$ and, by symmetry, we get $\left[i_{\ell}\right]-\left[i_{\ell^{-1}}\right] \leq 0$. This shows that $\left[i_{\ell}\right]=\left[i_{\ell^{-1}}\right]$ if $P$ satisfies the hard Lefschetz property (8). The result is shown similarly if $P$ satisfies the hard Lefschetz property (9).

We get the converse going backward, applying the hard Lefschetz theorem for the cohomology of $\mathcal{X}_{\ell}$, see for instance [3, Theorem 12.5.8 and (12.5.2)] where $\omega$ is the cohomology class of an ample divisor (by [2. Proposition 4.7], its restrictions to the twisted sectors are also ample because a strictly convex function descends to a a strictly convex function on the quotient fan, see for instance [9, p. 12]).

Remark 3.6 Theorem 3.5 has been suggested by 8 . If $P$ is reflexive, the ages are integers and $P$ satisfies $(H L)$ if and only if age $\left(\mathcal{X}_{\ell}\right)=\operatorname{age}\left(\mathcal{X}_{\ell^{-1}}\right)$. This result is already stated in loc. cit.

Remark 3.7 Assume that the toric variety $X_{\Sigma_{P}}$ is smooth: we have $\mathcal{X}_{P}=X_{\Sigma_{P}}$ and equality (10) holds true since there are no twisted sectors. This matches with [17, Proposition 3.4].

Corollary 3.8 If $f_{P}$ satisfies $(H L)$ if and only if $\left[\operatorname{age}\left(\mathcal{X}_{\ell}\right)\right]=\left[\operatorname{age}\left(\mathcal{X}_{\ell^{-1}}\right)\right]$ for all $\ell \in F$. 
Proof. Assume first that $f_{P}$ satisfies (HL). We use Proposition 3.2 and Theorem 3.5 in order to get the conditions on the ages. Conversely, the equality of the ages shows that the hard Lefschetz property hold for $P$ (again by Theorem [3.5) and by [2, Lemma 5.1] the preimage of $f_{P}$ under the mirror isomorphism $\varphi$ of Proposition 3.2 is the cohomology class of the $\mathbb{Q}$-ample divisor $\sum_{i=1}^{r} \ell_{i}^{-1} D_{i}$ where the positive integer $\ell_{i}$ is defined by $b_{i}=\ell_{i} a_{i}, a_{i}$ denoting the primitive lattice generator of the ray $\rho_{i}$ of the fan $\Sigma_{P}$.

Fortunately, condition (10) has an easy combinatorial description. Let $\Sigma_{P}=\left(\mathbb{Z}^{n}, \Sigma_{P}, \mathcal{V}(P)\right)$ be the stacky fan of $P$. For $\sigma$ a $n$-dimensional cone in the fan $\Sigma_{P}$, we denote by $\operatorname{Box}(\sigma)$ the set of the elements $v \in N$ such that $v=\sum_{\rho_{i} \subseteq \sigma} q_{i} b_{i}$ for some $0 \leq q_{i}<1$ where $\rho_{i}$ is the ray generated by the vertex $b_{i}$ of $P$. Let $\operatorname{Box}\left(\Sigma_{P}\right)$ be the union of $\operatorname{Box}(\sigma)$ for all $n$-dimensional cones $\sigma \in \Sigma_{P}$.

Proposition 3.9 Condition (10) holds true if and only if

$$
[\nu(v)]=(\operatorname{dim} \sigma(v)-1) / 2 \text { if } \nu(v) \notin \mathbb{N}
$$

and

$$
\nu(v)=\operatorname{dim} \sigma(v) / 2 \text { if } \nu(v) \in \mathbb{N}
$$

for all $v \in \operatorname{Box}\left(\boldsymbol{\Sigma}_{P}\right)$, where $\sigma(v)$ the smallest cone of $\Sigma_{P}$ containing $v$ and $\nu$ is the Newton function of $P$.

Proof. By [2, Proposition 4.7], the sectors $\mathcal{X}_{v}$ are parametrized by $v \in \operatorname{Box}\left(\boldsymbol{\Sigma}_{P}\right)$ and $\operatorname{dim} \mathcal{X}_{v}=n-\operatorname{dim} \sigma(v)$. Let $v \in \operatorname{Box}\left(\boldsymbol{\Sigma}_{P}\right)$. Because $\operatorname{dim} \mathcal{X}_{v}=n-\operatorname{age}\left(\mathcal{X}_{v}\right)-\operatorname{age}\left(\mathcal{X}_{v^{-1}}\right)$, we get age $\left(\mathcal{X}_{v}\right)+\operatorname{age}\left(\mathcal{X}_{v^{-1}}\right)=\operatorname{dim} \sigma(v)$. Therefore, $\left[\operatorname{age}\left(\mathcal{X}_{v}\right)\right]+\left[\operatorname{age}\left(\mathcal{X}_{v^{-1}}\right)\right]=\operatorname{dim} \sigma(v)-1$ if age $\left(\mathcal{X}_{v}\right) \notin \mathbb{N}$ and $\left[\operatorname{age}\left(\mathcal{X}_{v}\right)\right]+\left[\operatorname{age}\left(\mathcal{X}_{v^{-1}}\right)\right]=\operatorname{dim} \sigma(v)$ if $\operatorname{age}\left(\mathcal{X}_{v}\right) \in \mathbb{N}$. Because age $\left(\mathcal{X}_{v}\right)=\nu(v)$ by [2, Remark 5.4], the result follows from Theorem 3.5 .

This is Theorem 1.1 in the introduction.

\section{Application to simplices}

We apply the previous results to simplices and we deduce some consequences in Hodge theory.

\subsection{Hard Lefschetz property for simplices}

In this text, we will say that the polytope $\Delta:=\operatorname{conv}\left(v_{0}, \cdots, v_{n}\right)$ is a simplex if its vertices $v_{i}$ belong to the lattice $\mathbb{Z}^{n}$ and if it contains the origin as an interior point. The weight of a simplex $\Delta$ is the tuple $Q(\Delta)=\left(q_{0}, \cdots, q_{n}\right)$ where

$$
q_{i}:=\left|\operatorname{det}\left(v_{0}, \cdots, \widehat{v_{i}}, \cdots, v_{n}\right)\right|
$$

for $i=0, \cdots, n$. We will always assume that the tuple $Q(\Delta)=\left(q_{0}, \cdots, q_{n}\right)$ is arranged by increasing order (this can always be achieved by renumbering the vertices) and we will put $\mu:=q_{0}+\cdots+q_{n}$. The simplex $\Delta$ is reduced if $\operatorname{gcd}\left(q_{0}, \cdots, q_{n}\right)=1$ (see [4]). Up to unimodular transformations, there exists a unique reduced simplex $\Delta$ of weight $\left(q_{0}, q_{1}, \cdots, q_{n}\right)$ and an algorithm in order to construct it, and therefore to get $f_{\Delta}$, is given in [4, Theorem 3.6] (recall that $f_{\Delta}$ denotes the Laurent polynomial defined by $f_{\Delta}(u)=\sum_{i=0}^{n} u^{v_{i}}$ on $\left.\left(\mathbb{C}^{*}\right)^{n}\right)$.

By Remark 3.7, the hard Lefschetz property is true if $\left(q_{0}, \cdots, q_{n}\right)=(1, \cdots, 1)$. We now give a criterion about the remaining cases. Let $\Delta$ be a simplex of weight $\left(q_{0}, \cdots, q_{n}\right)$. We define

$$
F:=\left\{\frac{\ell}{q_{i}} \mid 0 \leq \ell \leq q_{i}-1,0 \leq i \leq n\right\} .
$$

We will denote by $f_{1}, \cdots, f_{k}$ the elements of $F$ arranged by increasing order and we will put

$$
d_{i}:=\operatorname{Card}\left\{j \mid q_{j} f_{i} \in \mathbb{Z}\right\} .
$$

We have $f_{1}=0$ and $d_{1}=n+1$. 
Proposition 4.1 Let $\Delta$ be a reduced simplex of weight $\left(q_{0}, \cdots, q_{n}\right)$ such that $q_{n} \geq 2$. Then $\Delta$ satisfies (HL) if and only if $f_{\Delta}$ satisfies $(H L)$. And this happens if and only if

$$
\left[-\mu f_{i}+\sum_{\ell=1}^{i-1} d_{\ell}\right]=\frac{d_{1}-d_{i}-1}{2} \text { for } i \geq 2
$$

if $-\mu f_{i}+\sum_{\ell=1}^{i-1} d_{\ell} \notin \mathbb{Z}$ and

$$
-\mu f_{i}+\sum_{\ell=1}^{i-1} d_{\ell}=\frac{d_{1}-d_{i}}{2} \text { for } i \geq 2
$$

if $-\mu f_{i}+\sum_{\ell=1}^{i-1} d_{\ell} \in \mathbb{Z}$.

Proof. The first assertion follows from Corollary 3.8. According to [6, Section 3.4], the sectors of $\mathcal{X}_{\Delta}$ are labelled by the set $F$ and the ages of the sectors $\mathcal{X}_{f_{\ell}}$ are age $\left(\mathcal{X}_{f_{1}}\right)=0$ and age $\left(\mathcal{X}_{f_{i}}\right)=\sum_{\ell=1}^{i-1} d_{\ell}-\mu f_{i}$ if $i=2, \cdots, k$. By the proof of Proposition [3.9, (HL) holds if and only if $2\left[\operatorname{age}\left(\mathcal{X}_{v}\right)\right]=n-1-\operatorname{dim} \mathcal{X}_{v}$ if $\operatorname{age}\left(\mathcal{X}_{v}\right) \notin \mathbb{N}$ and $2 \operatorname{age}\left(\mathcal{X}_{v}\right)=n-\operatorname{dim} \mathcal{X}_{v}$ if age $\left(\mathcal{X}_{v}\right) \in \mathbb{N}$. This gives the remaining assertions because $\operatorname{dim} \mathcal{X}_{f_{i}}=d_{i}-1$ and $d_{1}=n+1$.

Example 4.2 We give here two basic examples.

1. Let $\Delta$ be the reduced simplex of weight $Q(\Delta)=(1,1,3)$. Then $f_{1}=0, f_{2}=1 / 3, f_{3}=2 / 3, d_{1}=3$, $d_{2}=1, d_{3}=1$ and $\mu=5$ : the simplex $\Delta$ does not satisfy $(H L)$.

2. Let $\Delta$ be the reduced simplex of weight $Q(\Delta)=(1,2,2,3)$, for which $\mu=8$. Then $f_{1}=0, f_{2}=1 / 3$, $f_{3}=1 / 2, f_{4}=2 / 3, d_{1}=4, d_{2}=1, d_{3}=2, d_{4}=1$ : the simplex $\Delta$ satisfies (HL).

Recall that the reduced simplex $\Delta$ of weight $\left(q_{0}, \cdots, q_{n}\right)$ is reflexive if and only if $q_{i}$ divides $\mu$ for $i=0, \cdots, n$, see [4, Proposition 5.1].

Corollary 4.3 A reduced and reflexive simplex $\Delta$ of weight $\left(q_{0}, \cdots, q_{n}\right)$ with $q_{n} \geq 2$ satisfies (HL) if and only if

$$
-\mu f_{i}+\sum_{\ell=1}^{i-1} d_{\ell}=\left(d_{1}-d_{i}\right) / 2
$$

for $i=2, \cdots, k$.

Remark 4.4 Assume that the reduced and reflexive simplex $\Delta$ satisfies (HL). Then, if $q_{n} \geq 2$, it follows from Corollary 4.3 that we must have

$$
\frac{2 \mu}{q_{n}}=n+1+m\left(q_{n}\right)
$$

where $m\left(q_{n}\right)$ denotes the multiplicity of $q_{n}$ in the tuple $\left(q_{0}, \cdots, q_{n}\right)$ because $f_{2}=1 / q_{n}$ and $d_{2}=m\left(q_{n}\right)$ (recall that we assume that the tuple $\left(q_{0}, \cdots, q_{n}\right)$ is arranged by increasing order): this is Proposition 1.2 in the introduction. Most of the time it will be enough to notice that this necessary condition does not hold in order to show that the hard Lefschetz condition (HL) fails for $\Delta$.

Example 4.5 Reduced and reflexive simplices are classified up to dimension four in [4]. Using Corollary 4.3 and Remark 4.4, we get the following statements:

- two dimensional reduced and reflexive simplices satisfy the hard Lefschetz property;

- if $n=3$, there are 14 reduced and reflexive simplices (up to unimodular transformations) and the hard Lefschetz property hold only for the simplices with weights $(1,1,1,1)$ and $(1,1,2,2)$. 
- if $n=4$, there are 147 reduced and reflexive simplices (up to unimodular transformations) and the hard Lefschetz property hold only for the simplices $\Delta$ with weights $(1,1,1,1,1),(1,1,1,1,2),(1,1,2,2,2)$, $(1,2,3,3,3)$ and $(1,2,2,3,4)$.

Of course, our results apply to greater dimensions: for instance, it is immediately seen the reduced and reflexive simplex of weight $(1,1,1,1,1,1,3)$ in $\mathbb{R}^{6}$ does not satisfy the hard Lefschetz property.

\subsection{Application to Hodge theory for reflexive simplices}

We keep in this section the setting and the notations of [17. Let $f$ be a convenient and nondegenerate Laurent polynomial on $\left(\mathbb{C}^{*}\right)^{n}$ and let $P$ be its Newton polytope. It is known that $f$ defines a mixed Hodge structure $M H S_{f}:=\left(H, F^{\bullet} H, W \bullet H\right)$ and this mixed Hodge structure is said to be of Hodge-Tate type if

1. $W_{2 i+1} H=W_{2 i} H$ for $i \in \mathbb{Z}$,

2. the filtrations $F^{\bullet} H$ and $W_{2}$ are opposite, that is $\operatorname{gr}_{F}^{p} \operatorname{gr}_{2 q}^{W} H=0$ for $p \neq q$.

The link with the hard Lefschetz property is given by the following result:

Proposition 4.6 [17, Corollary 2.6] The following are equivalent:

1. the mixed Hodge structure $M H S_{f}$ is of Hodge-Tate type,

2. $f$ satisfies the hard Lefschetz property of Definition 3.1.

When $P$ is reflexive, we will say that $f$ satisfies the $K K P$ conjecture if $\operatorname{dim} \operatorname{gr}_{F}^{p} H=\operatorname{dim} \operatorname{gr}_{2 p}^{W} H$ (see [12, Conjecture 3.6], but also [17, 3.a] and [18]). We keep the notations of Section 4.1]

Proposition 4.7 Let $\Delta$ be a reduced and reflexive simplex in $\mathbb{R}^{n}$ with weight $Q(\Delta)=\left(q_{0}, \cdots, q_{n}\right)$, where $q_{n} \geq 2$. The Laurent polynomial $f_{\Delta}$ satisfies the KKP conjecture if and only if

$$
\sum_{\ell=1}^{i-1} d_{\ell}-\mu f_{i}=\frac{d_{1}-d_{i}}{2}
$$

for $i=2, \cdots, k$.

Proof. By [17, Lemma 2.4 and Corollary 2.6], $f$ satisfies the KKP conjecture if and only if $f$ satisfies (HL). Thus, the result follows from Proposition 4.1 and Corollary 4.3 .

If $\Delta$ is a reduced and reflexive simplex in $\mathbb{R}^{n}$ for $n=2,3$, we get from Example 4.5 the Laurent polynomials $f_{\Delta}$ which satisfy the KKP conjecture.

\section{Application to the distribution of spectral numbers}

We apply the previous results to the study of the distribution of the spectrum at infinity of a convenient and nondegenerate Laurent polynomial $f$ defined on $\left(\mathbb{C}^{*}\right)^{n}$. Recall that a polynomial $a_{0}+a_{1} z+\cdots+a_{n} z^{n}$ is unimodal if there exists an index $j$ such that $a_{i} \leq a_{i+1}$ for all $i<j$ and $a_{i} \geq a_{i+1}$ for all $i \geq j$. 


\subsection{Unimodality of the spectrum at infinity: unipotent case}

We study in this section the unimodality of the spectrum at infinity if $f$ satisfies the assumption of Corollary 2.2 . So let us assume that

$$
\operatorname{Spec}_{f}(z)=1+d(1) z+\cdots+d(n-1) z^{n-1}+z^{n}
$$

where $d(i):=\operatorname{dim}_{\mathbb{C}} \operatorname{gr}_{i}^{\mathcal{N}} \mathcal{A}_{f}$ for $i=1, \cdots, n-1$. The results in this section follow from well-known in combinatorics. The first one is due to Hibi [10]:

Proposition 5.1 We have $1 \leq d(1) \leq d(i)$ for $i \leq[n / 2]$. In particular, $\operatorname{Spec}_{f}(z)$ is unimodal if $n \leq 5$.

Proof. Let $P$ be the Newton polytope of $f$ and let $\delta_{P}(z)=\delta_{0}+\delta_{1} z+\cdots+\delta_{n} z^{n}$ be its $\delta$-vector. By Corollary 2.2 and [5, Corollary 2.2], $P$ is reflexive and $\operatorname{Spec}_{f}(z)=\delta_{0}+\delta_{1} z+\cdots+\delta_{n} z^{n}$. By [10], we have $\delta_{0} \leq \delta_{1} \leq \delta_{j}$ for $2 \leq j \leq[n / 2]$. The inequalities follow and we use then the symmetry $d(i)=d(n-i)$ in order to get the unimodality for $n \leq 5$.

Nevertheless, in this situation the spectrum at infinity needs not to be unimodal if $n \geq 6$. The following counter-example is provided by [16]:

Proposition 5.2 Let $s \geq 2, k \geq 2$ be two integers and let $n:=$ sk. Let $f_{\Delta}$ be the Laurent polynomial defined by

$$
f_{\Delta}\left(u_{1}, \cdots, u_{n}\right):=u_{1}+\cdots+u_{n}+\frac{1}{u_{1} \cdots u_{n-1} u_{n}^{s}}
$$

on $\left(\mathbb{C}^{*}\right)^{n}$. Then,

1. $f_{\Delta}$ is convenient and nondegenerate,

2. the Milnor number of $f_{\Delta}$ is equal to $s(k+1)$,

3. $\operatorname{Spec}_{f_{\Delta}}(z)=1+z+\cdots+z^{s k}+z^{(s-1) k}+z^{(s-2) k}+\cdots+z^{k}$,

4. the spectrum at infinity of $f_{\Delta}$ is unimodal if and only if $s=2$,

5. $f_{\Delta}$ satisfies the hard Lefschetz property if and only if $s=2$.

Proof. Let $\Delta:=\operatorname{conv}\left(e_{1}, \cdots, e_{n},-\sum_{i=1}^{n} q_{i} e_{i}\right)$ where $n:=s k,\left(e_{1}, \cdots, e_{n}\right)$ is the canonical basis of $\mathbb{R}^{n}$ and $\left(q_{1}, \cdots, q_{n}\right):=(1, \cdots, 1, s)$ where 1 is counted $s k-1$-times. The simplex $\Delta$ is reduced and reflexive and is the Newton polytope of $f_{\Delta}$. Its weight is $\left(q_{0}, q_{1}, \cdots, q_{n}\right)=(1, \cdots, 1, s)$ where 1 is counted $s k$ times and $\mu_{\Delta}=s(k+1)$. The nondegeneracy follows from the fact that the facets of $\Delta$ are simplices. The assertion on the Milnor number follows from [13. Using the results recalled in Section 4.1, we get $f_{1}=0, f_{2}=1 / s, \cdots, f_{s}=(s-1) / s, d_{1}=n+1, d_{2}=\cdots=d_{s}=1$. Define $\beta_{1}:=0$ and

$$
\beta_{i}:=d_{1}+\cdots+d_{i-1}-\mu f_{i}=k(s-(i-1))
$$

for $i=2, \cdots, s$. By [6], the spectrum at infinity of $f_{\Delta}$ is given by $\beta_{1}, \beta_{1}+1, \cdots, \beta_{1}+d_{1}-1, \cdots, \beta_{k}, \beta_{k}+$ $1, \cdots, \beta_{k}+d_{k}-1$, and the formula for $\operatorname{Spec}_{f_{\Delta}}(z)$ follows. The assertion about unimodality is clear and for the last statement, notice that the necessary and sufficient condition of Corollary 4.3 is $s=2(i-1)$ for $i=2, \cdots, s$ and is satisfied only for $s=2$.

Remark 5.3 Because $\Delta$ is reflexive, $\operatorname{Spec}_{f_{\Delta}}(z)$ is equal to the $\delta$-vector of $\Delta$, see Proposition 2.1. This formula for the $\delta$-vector of $\Delta$ can already be found in [16].

If $n \geq 6$, we have the following positive result: 
Proposition 5.4 Let $f$ be a convenient and nondegenerate Laurent polynomial on $\left(\mathbb{C}^{*}\right)^{n}$ whose spectrum at infinity is a polynomial. Assume that $f$ satisfies the hard Lefschetz property of definition 3.1. Then $\operatorname{Spec}_{f}(z)$ is unimodal.

Proof. The hard Lefschetz property shows that $[f]: \operatorname{gr}_{i-1}^{\mathcal{N}} \mathcal{A}_{f} \longrightarrow \operatorname{gr}_{i}^{\mathcal{N}} \mathcal{A}_{f}$ is injective for $i \leq n / 2$ and surjective for $i>n / 2$.

Of course, the converse is not true: by Proposition [5.1. $\operatorname{Spec}_{f_{\Delta}}(z)$ is unimodal for any four dimensional reduced and reflexive simplex $\Delta$ but $f_{\Delta}$ does not satisfy the hard Lefschetz property in general (see Example 4.5).

\subsection{Unimodality of the spectrum at infinity: the general case}

We consider now the general case, that is when the spectrum at infinity of $f$ is not necessarily a polynomial: we write

$$
\operatorname{Spec}_{f}(z)=\sum_{i} d\left(\alpha_{i}\right) z^{\alpha_{i}}
$$

where $d\left(\alpha_{i}\right):=\operatorname{dim}_{\mathbb{C}} \operatorname{gr}_{\alpha_{i}}^{\mathcal{N}} \mathcal{A}_{f}$ and $\alpha_{i} \in \mathbb{Q}$, the rational numbers $\alpha_{i}$ being arranged by increasing order. Using the symmetry property $z^{n} \operatorname{Spec}_{f}\left(z^{-1}\right)=\operatorname{Spec}_{f}(z)$, one would expect that

$$
d\left(\alpha_{1}\right) \leq d\left(\alpha_{2}\right) \leq \cdots \leq d\left(\alpha_{\ell}\right)
$$

for all $\alpha_{\ell} \leq n / 2$. Unfortunately, and unlike Section 5.1 this may fail if $n \leq 5$ or if $f$ satisfies the hard Lefschetz property (HL) (see example 5.6 below).

So what gives in this case this hard Lefschetz property? Let us write $\operatorname{Spec}_{f}(z)=\sum_{\alpha \in[0,1[} z^{\alpha} \operatorname{Spec}_{f}^{\alpha}(z)$ where $\operatorname{Spec}_{f}^{\alpha}(z) \in \mathbb{Q}[z]$.

Proposition 5.5 Assume that $f$ satisfies the hard Lefschetz property of definition 3.1. Then the polynomials $\operatorname{Spec}_{f}^{\alpha}(z)$ are unimodal for $\alpha \in[0,1[$.

Proof. The hard Lefschetz assumption shows that $[f]: \operatorname{gr}_{\alpha+i-1}^{\mathcal{N}} \mathcal{A}_{f} \longrightarrow \operatorname{gr}_{\alpha+i}^{\mathcal{N}} \mathcal{A}_{f}$ is injective for $i \leq(n-1) / 2$ and surjective for $i>(n-1) / 2$ for $\alpha \in] 0,1[$. The case $\alpha=0$ has been considered in Proposition [5.4

Example 5.6 Let $f$ be the Laurent polynomial defined by $f\left(u_{1}, u_{2}, u_{3}\right)=u_{1}+u_{2}+u_{3}+1 / u_{1}^{2} u_{2}^{2} u_{3}^{3}$ on $\left(\mathbb{C}^{*}\right)^{3}$. Then $\operatorname{Spec}_{f}(z)=1+2 z+z^{4 / 3}+z^{5 / 3}+2 z^{2}+z^{3}$ and does not satisfy (14). However, $f$ satisfies (HL) (see Example 4.2). We have $\operatorname{Spec}_{f}^{0}(z)=1+2 z+2 z^{2}+z^{3}, \operatorname{Spec}_{f}^{1 / 3}(z)=z, \operatorname{Spec}_{f}^{2 / 3}(z)=z$ and these polynomials are unimodal.

\section{References}

[1] Adem, A., Leida, J., Ruan, Y.: Orbifolds and stringy topology, Cambridge tracts in Math., 171, 2007.

[2] Borisov, L., Chen, L., Smith, G.: The orbifold Chow ring of toric Deligne-Mumford stacks, J. Amer. Soc. , 18 (1), 2005, p. 193-215.

[3] Cox, D., Little, J., Schenck, A.: Toric varieties, American Mathematical Society, 124, 2010.

[4] Conrads, H. : Weighted projective spaces and reflexive simplices, Manuscripta Math., 107, 2002, p. 215-227.

[5] Douai, A.: Ehrhart polynomials of polytopes and spectrum at infinity of Laurent polynomials, J. Alg. Comb. (2020). https://doi.org/10.1007/s10801-020-00984-x 
[6] Douai, A., Mann, E.: The small quantum cohomology of a weighted projective space, a mirror D-module and their classical limits, Geometriae Dedicata, 164, 2013, p. 187-226.

[7] Douai, A., Sabbah, C.: Gauss-Manin systems, Brieskorn lattices and Frobenius structures II, In : Frobenius Manifolds, C. Hertling and M. Marcolli (Eds.), Aspects of Mathematics E 36, 2004.

[8] Fernandez, J.: Hodge structures for orbifold cohomology, Proc. Amer. Math. Soc., 134 (9), 2006, p. 2511-2520.

[9] Gross, M.: Tropical Geometry and Mirror Symmetry, CBMS Regional Conf. Ser. in Math., vol. 114, AMS, Providence, 2011.

[10] Hibi, T.: Algebraic Combinatorics on Convex Polytopes, Carslaw Publications, Australia, 1992.

[11] Hibi, T.: Dual polytopes of rational convex polytopes, Combinatorica, 12 (2), 1992, p. 237-240.

[12] Katzarkov, L., Kontsevich, M., Pantev, T.: Bogomolov-Tian-Todorov theorems for Landau-Ginzburg models, J. Diff. Geom., 105 (1), 2017, p. 55-117.

[13] Kouchnirenko, A.G.: Polyèdres de Newton et nombres de Milnor, Invent. Math., 32, 1976, p. 1-31.

[14] Mann, E.: Orbifold quantum cohomology of weighted projective spaces, Journal of Algebraic Geometry, 17, 2008, p. 137-166.

[15] Mustaţă, M., Payne, S. : Ehrhart polynomials and stringy Betti numbers, Math. Ann., 333 (4), 2005, p. 787-795.

[16] Payne, S.: Ehrhart series and lattice triangulations, Discrete Comput. Geom., 40 (3), 2008, p. 365-376.

[17] Sabbah, C.: Some properties and applications of Brieskorn lattices, Journal of Singularity theory, 18, 2018, p. 238-247.

[18] Shamoto, Y.: Hodge-Tate conditions for Landau-Ginzburg models, arXiv:1709.03244.

[19] Stanley, R.: Combinatorial applications of the hard Lefschetz theorem, in Proc. of the International Congress of Mathematicians (Warsaw, 1983), North-Holland, Amsterdam, 1984, p. 447-453.

[20] Stanley, R.: A monotonicity porperty of h-vectors and $h^{*}$-vectors, Europ. J. Combinatorics, 14, 1993, p. 251-258. 